\title{
3D Model of the Heart Electrical Activity With Heterogeneous Ventricular Action Potentials
}

\author{
Niccoló Biasi ${ }^{1}$, Alessandro Tognetti ${ }^{1,2}$ \\ ${ }^{1}$ Dipartimento di Ingegneria dell'Informazione, Universitá di Pisa, Italy \\ ${ }^{2}$ Centro E.Piaggio, Universitá di Pisa, Italy
}

\begin{abstract}
We developed a three-dimensional finite element model of the electrical activity of the whole heart embedded in the torso that is proposed as an useful tool to simulate ECG signals both in healthy and pathological conditions. The electrical activity of the cardiac tissue is reproduced with the well-known bidomain model. The ionic current dynamics is represented with a modified version of the FitzHughNagumo model. The model generates a controlled spontaneous activation in the sinoatrial node. The model reproduce realistic electrocardiographic signals both in healthy and pathological conditions (e.g. ischemia).
\end{abstract}

\section{Introduction}

Mathematical modelling of electrical activity of the heart is a growing field in modern cardiac research. Models of the electrical activity of the heart are important tools for understanding cardiac functions and diseases. In addition, they can be employed for the simulation and design of stimulating devices such as pacemakers and defibrillators. Several works have focused on the development of mathematical models of the heart, e.g. [1-4]. Most of the existing models are too complex, include only atria or ventricles or avoid modelling the tissues surrounding the heart.

In this work, we present the development of a whole heart-torso model aimed at electrocardiogram (ECG) simulation in healthy and pathological conditions. Our model of the electrical activity of the heart is based on the bidomain theory coupled with modified FitzHugh-Nagumo (FHN) phenomenological model for the computation of the action potentials (APs) in the different heart regions. Compared to our previous work [5], we introduced the heterogeneous characteristics of the ventricular APs. In particular, we added the ventricular midcardium by modeling APs differently than in the epicardial and endocardial regions. Furthermore, we added the description of transient outward current and improved the quality of the simulated APs through the introduction of a new shape parameter.
Our model is able to generate realistic healthy and pathological ECGs. The addition of the ventricular midcardium and the new characteristics of the cell model have improved the quality of the generated ECG which presented a more realistic T-wave. Indeed, unrealistic T-wave was the main flaw of our previous work reported in [5].

As example of pathological condition, we simulated anterior subepicardial ischemia. To reproduce the ischemic AP we simply adjust some model parameters.

\section{Methods}

We developed a 3D model describing the electrical activity of the heart composed by different regions defining torso, lungs, myocardium and cardiac chambers (Fig. 1). We implemented the model in finite elements (FE) through Comsol Multiphysics (version 5.4, COMSOL AB, Stockhlom, Sweden). As described in Fig. 2, we divided the myocardium in different sub-domains describing the sinoatrial node, atria, atrioventricular node, His bundle, bundle branches, endocardium, midcardium and epicardium.

Our model is based on the bidomain theory in which the heart is represented as the coupling of the intracellular

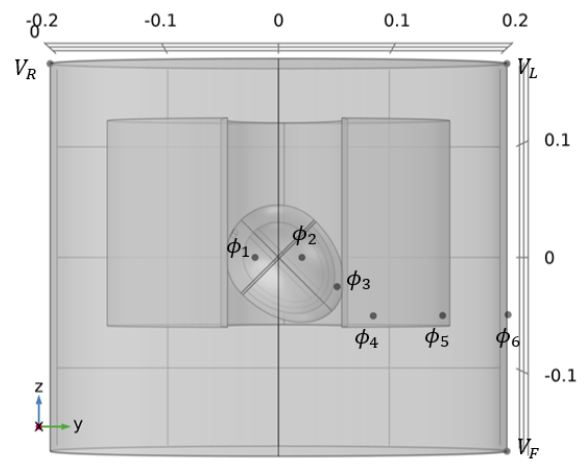

Figure 1. Model geometry observed in the frontal plane. The labelled points represent the probes used to simulate ECG. The measurement unit is meter 
and extracellular continuous domains. According to the bidomain theory, intracellular $\left(\phi_{i}\right)$ and extracellular $\left(\phi_{o}\right)$ potentials are defined in each point of the heart volume and are coupled by the cell membrane through the membrane current. The membrane potential $V_{M}$ is defined as the difference between $\phi_{i}$ and $\phi_{o}$. As described in [5], by applying the bidomain theory we obtain:

$$
\begin{gathered}
\xi \frac{\partial \phi_{i}}{\partial t}-\xi \frac{\partial \phi_{o}}{\partial t}-\nabla \cdot\left(\xi k_{i} \nabla \phi_{i}\right)=-\xi I_{i o n} \\
\xi \frac{\partial \phi_{o}}{\partial t}-\xi \frac{\partial \phi_{i}}{\partial t}-\nabla \cdot\left(\xi k_{o} \nabla \phi_{o}\right)=\xi I_{i o n}
\end{gathered}
$$

where $k_{i}=\frac{\sigma_{i}}{C_{M}}, k_{o}=\frac{\sigma_{o}}{C_{M}}$ and $I_{\text {ion }}$ is the ionic current per unit of membrane capacitance $\left(C_{M}\right)$. The $\xi$ parameter regulates the density of myocytes inside specific regions of the myocardium and can be modified to regulate the amplitude of the single waves of the ECG.

For the definition of $I_{\text {ion }}$ we adopted the FHN phenomenological model [6]. With respect to our previous work we added the transient outward current in the definition of $I_{i o n}$ for the ventricular regions (i.e. the His bundle, the bundle branches and the myocardial wall), by means of a new variable $w$ :

$$
\begin{aligned}
I_{\text {ion }}= & k g c_{1}\left(V_{M}-B\right)\left(a-\frac{V_{M}-B}{A}\right)\left(1-\frac{V_{M}-B}{A}\right) \\
& +k g c_{2} u\left(V_{M}-B\right)+k g c_{3} w\left(V_{M}-B\right)(1-d u)
\end{aligned}
$$

$w$ is defined by the following equation:

$$
\frac{\partial w}{\partial t}= \begin{cases}k e_{w^{+}}\left(\frac{V_{M}-B}{A}-d_{w} w\right) & \text { if } \quad \frac{\partial w}{\partial t} \geq 0 \text { or } \\ -k e_{w^{-}} w \quad \text { otherwise } & V_{M} \leq B+5 m V\end{cases}
$$

The parameter $d_{w}$ regulates the strength of the outward transient current, while $e_{w^{+}}$and $e_{w^{-}}$regulate the raise and decay time of the variable $w$ and consequently of the transient outward current. We set $d_{w}$ equal to 1.2 in the epicardium, 1.3 in the midcardium and 1.4 in the other ventricular regions. $e_{w^{+}}$and $e_{w^{-}}$were set to 0.5 and 0.03 respectively in all the ventricular regions. We also introduced the new parameter $g$ which affects the shape of the $\mathrm{AP}$ and is defined piecewise:

$$
g= \begin{cases}g_{1} & \text { if } \quad \frac{\partial V_{M}}{\partial t}<0.1 \quad \text { and } \quad d_{w} w<0.5 \\ g_{2} & \text { otherwise }\end{cases}
$$

Also $c_{3}$ is defined piecewise to avoid that the transient outward current affects the phase 1 (i.e., the raise) of the AP:

$$
c_{3}= \begin{cases}c_{3}^{+} \quad \text { if } \quad \frac{\partial V_{M}}{\partial t} \geq 0 \text { and } \quad \frac{\partial w}{\partial t} \geq 0 \\ c_{3}^{-} \quad \text { otherwise }\end{cases}
$$

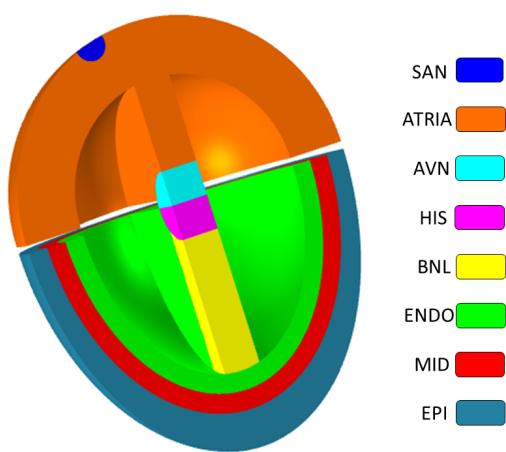

Figure 2. Heart subdomains.SAN:sinoatrial node, AVN:atrioventricular node, HIS:His bundle, BNL:bundle branches, ENDO:endocardium, MID:midcardium, EPI:epicardium

We set $g_{1}=0.5, g_{2}=1, c_{3}^{+}=0.1$ and $c_{3}^{-}=0.5 \mathrm{All}$ the other parameters and the refractoriness variable $u$ were already defined in [5]. In the supraventricular region we maintained the Rogers-McCulloch formulation (i.e. $g=1$ and $w=0$ ) [7]. In the sinoatrial node the ionic current was calculated through the classical rescaled FHN equation.

Ischemic AP is characterized by an increased resting potential and reduced action potential duration (APD) and upstroke [8-11]. Hence, we set $B=-65 \mathrm{mV}, A=$ $70 \mathrm{mV}$ and $e=0.008$ to reproduce this pathological condition. Furthermore, conduction velocity is also reduced, therefore we set the conductivity to 0.003 in the ischemic region. As example, we simulated anterior subepicardial ischemia.

The torso, the lungs and the cardiac chambers are considered passive conductors, so the governing equation is:

$$
\nabla \cdot(-\sigma \nabla V)=0
$$

where $\sigma$ is the tissue conductivity and $V$ is the potential in the passive volume conductors. The values of conductivity in the different tissues are the same used in [5]. All the external edges of the torso are considered electrically insulated. Instead, in all the edges connecting the heart and the passive conductors we assumed the continuity between the extracellular space and the passive conductors, while the intracellular space is considered electrically insulated from the external tissues. We placed nine probes on the torso surface (Fig. 1) to simulate the 12 standard ECG leads. In addition, we computed the heart vector defined by the resultant heart dipole moment and its location. In particular, the heart vector is obtained by minimizing the difference between the second order moments of the equivalent source (i.e., the heart dipole) and the real source (i.e., the dipole density distribution along the whole heart volume), as shown in [5]. 


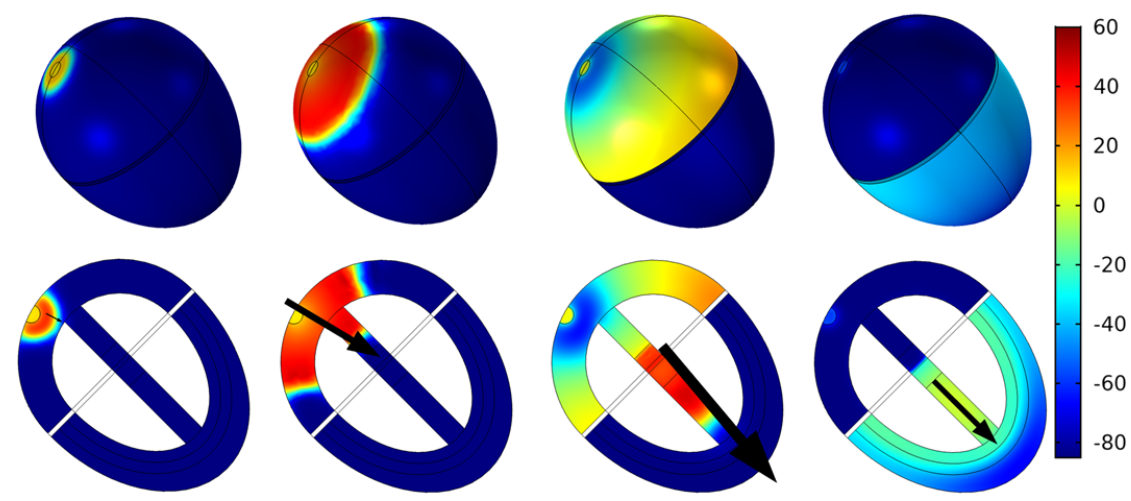

Figure 3. Membrane potential maps $(\mathrm{mV})$ and heart dipole in the principal steps of a cardiac cycle. First column: Spontaneous activation in the SAN. Second column: Atrial depolarization. Third column:Ventricular depolarization and atrial repolarization. Fourth column: Ventricular repolarization
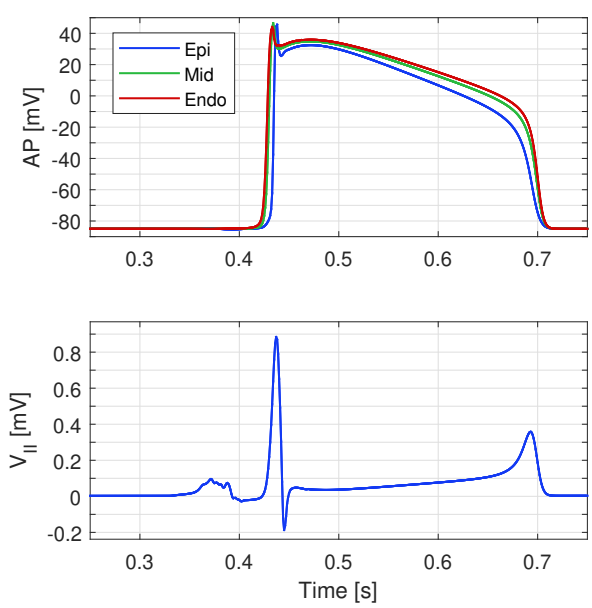

Figure 4. Healthy simulation. Top: Action potentials in the epicardium, midcardium and endocardium. Bottom: Second Eithoven lead

\section{Results}

Fig. 3 shows the membrane potential maps in the principal steps of a cardiac cycle spontaneously activated by the sinoatrial node. It also shows the equivalent dipole moment of the heart centered in its equivalent position. Note that the heart dipole has the same direction during depolarization and repolarization of the ventricles, resulting in same polarity of the QRS complex and T-wave. This is possible thanks to the heterogeneity of the AP in the three regions composing the ventricles, i.e. the epicardium, the midcardium and the endocardium (Fig. 4). In particular, the APD in the epicardial cells is lower than in the mid- cardium and endocardium. Even if the midcardium has been proved to contains M-cells with larger APD [12], we assumed that, on average, the endocardial AP is longer due to electrotonic coupling between adjacent cells. In fact, the endocardium is activated by the Purkinje fibers, which are characterized by very long APs, while the midcardial cells (i.e. the cells composing the midcardium together with the M-cells) have relatively short AP [13]. Our model can also reproduce heterogeneous AP notch, thanks to the introduction of the transient outward current. Indeed, the notch of the simulated AP is more prominent in the epicardial cells, as well known in cardiac electrophysiology [14]. The introduction of the new parameter $g$ also provides a better fit of the repolarization phase of the cardiac AP. As consequence, the simulated T-wave is smoother and more realistic with respect to our previous results. Fig. 6 shows the membrane potentials obtained in the epicardium in healthy and ischemic regions during simulation of subepicardial ischemia. In this condition, the heart dipoles are oriented inward of the ischemic region during the ST segment (Fig. 5). Indeed, the anterior ischemia is associated with ST elevation in the precordial leads (Fig. 6). This result is coherent with the diagnostic tools currently used in clinical practice [15].

\section{Conclusion}

We developed a finite element three-dimensional model of the electrical activity of the heart. Our model includes spontaneous activation in the sinoatrial node and is proposed as a tool to simulate ECG under both healthy and pathological conditions. Indeed, the model reproduces the main changes induced by ischemia on the ECG signals and 


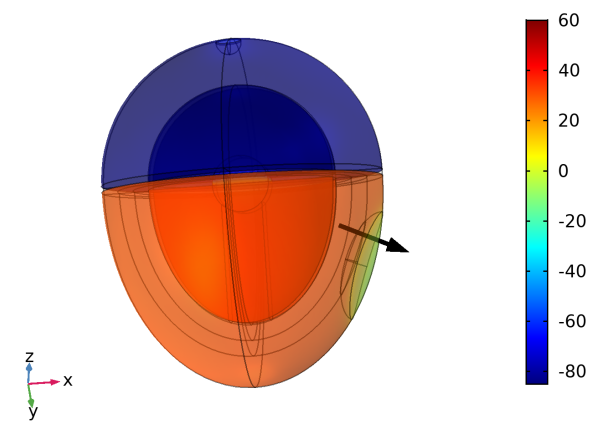

Figure 5. Potential map and heart dipole in presence of subepicardial ischemia during the ST interval
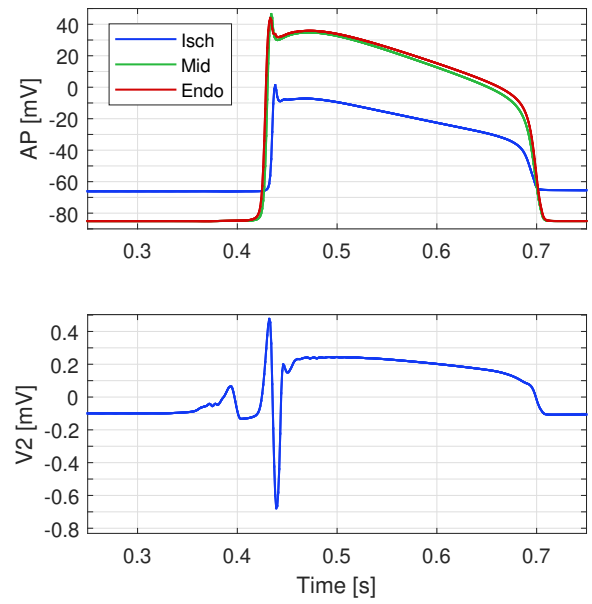

Figure 6. Ischemic simulation. Top: Action potentials in the ischemic region, midcardium and endocardium. Bottom: Second precordial lead

we believe it can be used to simulate also different conditions. Moreover, with the modifications introduced in this paper we add the description of transient outward current in our model and improve the quality of the ECG signal. In particular, the T-wave is more smoother and realistic. In addition, the developed model could be useful for educational and research purposes regarding the study of cardiac bioelectrical phenomena and their modifications induced by pathological conditions. A possible improvement of our model could be the use of anatomically realistic and patient-specific geometry with increased space resolution, including also anisotropic fiber conductivity.

\section{References}

[1] Trayanova NA. Whole-heart modeling: applications to cardiac electrophysiology and electromechanics. Circulation research 2011;108(1):113-128.
[2] Lines GT, Grottum P, Tveito A. Modeling the electrical activity of the heart: a bidomain model of the ventricles embedded in a torso. Computing and Visualization in Science 2003;5(4):195-213.

[3] Potse M. Scalable and accurate ecg simulation for reactiondiffusion models of the human heart. Frontiers in physiology 2018;9:370.

[4] Boulakia M, Fernández MA, Gerbeau JF, Zemzemi N. Numerical simulation of electrocardiograms. In Modeling of Physiological Flows. Springer, 2012; 77-106.

[5] Biasi N, Tognetti A. Modelling whole heart electrical activity for ischemia and cardiac pacing simulation. Health and Technology 2020;1-14.

[6] FitzHugh R. Impulses and physiological states in theoretical models of nerve membrane. Biophysical journal 1961; 1(6):445-466.

[7] Rogers JM, McCulloch AD. A collocation-galerkin finite element model of cardiac action potential propagation. IEEE Transactions on Biomedical Engineering 1994; 41(8):743-757.

[8] Pinto JM, Boyden PA. Electrical remodeling in ischemia and infarction. Cardiovascular research 1999;42(2):284297.

[9] Shaw RM, Rudy Y. Electrophysiologic effects of acute myocardial ischemia: a theoretical study of altered cell excitability and action potential duration. Cardiovascular research 1997;35(2):256-272.

[10] Janse M, Kleber AG. Electrophysiological changes and ventricular arrhythmias in the early phase of regional myocardial ischemia. Circulation Research 1981;49(5):10691081.

[11] Rodriguez B, Trayanova N, Noble D. Modeling cardiac ischemia. Annals of the New York academy of sciences 2006; 1080:395.

[12] Drouin E, Charpentier F, Gauthier C, Laurent K, Le Marec $\mathrm{H}$. Electrophysiologic characteristics of cells spanning the left ventricular wall of human heart: evidence for presence of $\mathrm{m}$ cells. Journal of the American College of Cardiology 1995;26(1):185-192.

[13] Stankovicova T, Szilard M, De Scheerder I, Sipido KR. M cells and transmural heterogeneity of action potential configuration in myocytes from the left ventricular wall of the pig heart. Cardiovascular research 2000;45(4):952-960.

[14] Nabauer M, Beuckelmann DJ, Uberfuhr P, Steinbeck G. Regional differences in current density and rate-dependent properties of the transient outward current in subepicardial and subendocardial myocytes of human left ventricle. Circulation 1996;93(1):168-177.

[15] Surawicz B, Knilans T. Chou's Electrocardiography in Clinical Practice E-Book: Adult and Pediatric. Elsevier Health Sciences, 2008.

Address for correspondence:

Niccoló Biasi

Scuola di Ingegneria - Largo Lucio Lazzarino, 2, 56122 Pisa

biasi.niccolo96@gmail.com 